\title{
EVIDÊNCIAS PALEOBOTÂNICAS DE INCÊNDIOS VEGETACIONAIS NO AFLORAMENTO MORRO PAPALÉO, PALEOZÓICO SUPERIOR DO RIO GRANDE DO SUL, BRASIL.
}

\author{
André Jasper ${ }^{1}$, Joseline Manfroi ${ }^{2}$, Elisa Ost Schmidt ${ }^{1}$, Neli Teresinha Galarce Machado ${ }^{1}$, \\ Odorico Konrad ${ }^{1} \&$ Dieter Uhl ${ }^{3}$
}

\begin{abstract}
RESUMO: Fragmentos de carvão vegetal macroscópico foram encontrados em um dos níveis fitofossilíferos do Afloramento Morro Papaléo, Paleozóico Superior (Asseliano/Sakmariano) da porção sul da Bacia do Paraná, Brasil. Foram descritos lenhos carbonizados, associados a dois morfotipos gimnospérmicos vinculados provavelmente a Agathoxylon, o que confirma a ocorrência de paleoincêndios vegetacionais nesta área. A deposição dos carvões vegetais macroscópicos se deu de forma autóctone/parautóctone, o que permite inferir que os paleoincêndios vegetacionais atingiram diretamente a turfeira formadora da camada de carvão e que ocorreram durante uma estação marcadamente seca. Correlações com estudos prévios indicam que os eventos tiveram como provável fonte de ignição atividades vulcânicas. A presença de carvão vegetal macroscópico em sedimentos permianos associados a diferentes níveis em localidades distintas, demonstra que incêndios vegetacionais foram comuns nos ambientes formadores dos depósitos do Paleozóico Superior da Bacia do Paraná.
\end{abstract}

Palavras-chave: carvão vegetal macroscópico; paleoincêndios; Gondwana; Permiano Inferior.

\begin{abstract}
PALEOBOTANICAL EVIDENCE OF WILDFIRES ON THE MORRO PAPALÉO OUTCROP, LATE PALEOZOIC OF RIO GRANDE DO SUL, BRAZIL. Macroscopic charcoal fragments have been discovered in one of the plant-bearing levels from the Morro Papaléo Outcrop, Late Paleozoic (Asselian/ Sakmarian) at the southernmost portion of the Paraná Basin, Brazil. Charred wood associated with two gymnospermic (probably Agathoxylon) morphotypes are described, confirming the occurrence of paleowildfires in this area. The macroscopic charcoal was autochthonously/parautochthonously deposited, allowing infers that the paleowildfires reached the mire which originated the coal layer and occurred during a dry season. Correlations with previous studies indicate that the events could maybe be ignited by volcanic activities. The presence of macroscopic charcoal in Permian sediments associated to different levels and localities, demonstrate that paleowildfires were more or less common in the environments associated with the Late Paleozoic deposits of the Paraná Basin.
\end{abstract}

Keywords: macroscopic charcoal; paleowildfires; Gondwana; Lower Permian.

\section{INTRODUÇÃO}

Em ambientes atuais, os incêndios vegetacionais são um importante elemento modelador nos mais diversos ecossistemas (Bowman et al., 2009; Flannigan et al., 2009). Além disso, está comprovado que este tipo de evento ocorria, com maior ou menor regularidade, nos mais variados sistemas desde o advento das plantas terrícolas (Glasspool et al., 2004).

De acordo com diferentes autores (Scott \& Stea, 2002; Preston \& Schmidt, 2006) o fogo, por ser um agente importante na dinâmica de um grande número de ecossistemas modernos, deve ter atuado de forma semelhante durante a história da Terra, tanto que, conforme Bond \& Keeley (2005) pode ser comparado à herbivoria em termos de impacto sobre a vegetação.

Evidências de paleoincêndios vegetacionais, em forma de charcoal (carvão vegetal) macroscópico fóssil [sensu Scott (2010) e, doravante, denominado de "carvão vegetal macroscópico" para fins de fluência do texto], podem ser encontradas em diferentes quantidades associadas às mais diversas idades geológicas, desde o Siluriano (Edwards \& Axe, 2004; Glasspool, et al., 2004) até o Quaternário (MacDonald et al., 1991; Scott, 1989; 2000; Scott \& Glasspool, 2006; Flannigan et al., 2009) o que confirma a afirmativa de Glasspool et al. (2004) sobre a sua freqüência desde o surgimento da vegetação terrícola.

1- Programa de Pós-Graduação em Ambiente e Desenvolvimento (PPGAD/UNIVATES).

Lajeado, Rio Grande do Sul, Brasil, CEP 95900-000.

2 - Setor de Botânica e Paleobotânica do Museu de Ciências Naturais da UNIVATES (SBP/MCN/UNIVATES). joselinemanfroi@universo.univates.br

3 - Senckenberg Forschungsinstitut und Naturmuseum, Senckenberganlage 25, 60325 Frankfurt am Main, Alemanha. Eberhard Karls Universität Tübingen, Institut für Geowissenschaften, Sigwartstraße 10, 72076 Tübingen, Alemanha. dieter.uhl@senckenberg.de. 
Cabe destacar que até recentemente, havia uma discrepância na quantidade de estudos sobre paleoincêndios vegetacionais evidenciados pelo registro de carvão vegetal macroscópico quando se compara diferentes regiões e idades da Terra ao longo do tempo geológico. Um exemplo disso é a diferença entre o número de estudos sobre este tipo de evento para o Paleozóico Superior. Havia um bom número de registros para o Hemisfério Norte, com estudos detalhados principalmente sobre a anatomia dos carvões vegetais para a Europa (Uhl \& Kerp, 2003; Uhl et al., 2004, 2008), América do Norte (Sander, 1987; Sander \& Gee, 1990; DiMichele et al., 2004) e China (Wang \& Chen, 2001). Enquanto isto, a quantidade de registros de mesma idade para o Hemisfério Sul, são bastante restritos e recentes (e.g. Jasper et al., 2008, 2011a, 2011b).

Outra discrepância pode ser observada quando se leva em consideração o registro de carvão vegetal macroscópico no Paleozóico Superior. Para o Intervalo Carbonífero/Permiano foram realizados trabalhos como os de Falcon-Lang (1998), Falcon-Lang \& Scott (2000, 2001), Uhl \& Kerp (2003) e Uhl et al. (2004, 2008). Já para o Triássico Inferior estes registros são inexistentes, fato que poderia estar relacionado, conforme alguns autores, à redução da concentração do $\mathrm{O}_{2}$ na atmosfera (Robinson, 1989; 1991; Scott, 2000).

Por outro lado, os registros de paleoincêndios vegetacionais, principalmente no que se refere às características paleobotânicas (e.g. anatomia, taxonomia e tafonomia dos carvões vegetais) do Paleozóico Superior do Gondwana ainda não foram estudados de forma aprofundada. Apenas algumas localidades foram avaliadas em detalhe quanto a este aspecto (Scott, 2000; Glasspool, 2000; Scott \& Glasspool, 2006; Jasper et al., 2007a, 2008, 2011a, 2011b; Uhl et al., 2008), de forma que uma boa parte desta área ainda pode ser considerada uma "região desconhecida" quando se trata da análise do combustível consumido durante esses eventos de paleoincêndios, o qual fica preservado sob a forma de carvão vegetal macroscópico.

No que diz respeito à análise das características paleobotânicas dos carvões vegetais do Paleozóico Superior do Gondwana e, consequentemente, de regiões mais específicas, como a Bacia do Paraná, os estudos dos paleoincêndios através desse tipo de registro é relativamente recente, com apenas alguns trabalhos publicados ou em análise. Isto gera a necessidade de reavaliações de localidades muitas vezes já amplamente estudadas sob outros aspectos, como ocorreu com o Afloramento Quitéria (Jasper et al., 2007a, 2008) e a Mina do Faxinal (Jasper et al., 2011b) ambos representativos da Formação Rio Bonito no Rio Grande do Sul.

Outra localidade para a qual apenas recentemente o registro de carvão vegetal macroscópico foi confirmado (Jasper et al., 2009) é o Afloramento Morro Papaléo. Situado no município de Mariana Pimentel, estado do Rio Grande do Sul, esta localidade tem idade Asseliana/ Sakmariana e expõe depósitos que representam o limite entre o Grupo Itararé e o Grupo Guatá, Formação Rio Bonito, Bacia do Paraná (Iannuzzi et al., 2006).

O Afloramento Morro Papaléo vem sendo estudado há mais de 40 anos, sendo conhecidos para esta localidade trabalhos de cunho paleobotânico (GuerraSommer \& Cazzulo-Klepzig, 1993; Vieira \& Iannuzzi, 2000; Iannuzzi et al., 2006; Souza \& Iannuzzi, 2007; Tybusch \& Iannuzzi, 2008), estratigráfico/ sedimentológico (Formoso, 1968; Piccoli et al., 1983; Lavina et al., 1992), palinológico (Corrêa da Silva \& Arrondo, 1977; Corrêa da Silva, 1978; CazzuloKlepzig et al., 1980; Souza \& Marques-Toigo, 2003, 2005; Smaniotto et al., 2006) e paleoecológicos/ paleogeográficos (Burjack et al., 1982; Paim et al., 1983 Pasqualini et al., 1986; Jasper et al., 2003, 2007a).

Todavia, estudos detalhados relativos a paleoincêndios e seu registro através de carvão vegetal macroscópico são inéditos para esta localidade. Desta forma, o presente trabalho apresenta o primeiro registro da ocorrência deste tipo de material e sua caracterização anatômica para um dos níveis pelíticos do Afloramento do Morro Papaléo, confirmando a manifestação de incêndios na paleovegetação do Permiano Superior da Bacia do Paraná a ele associada.

\section{CONTEXTO GEOLÓGICO E CARACTERIZAÇÃO DO AFLORAMENTO}

De acordo com Milani (2003), a Bacia do Paraná pode ser interpretada como uma bacia intracratônica de aproximadamente $1.400 .000 \mathrm{~km}^{2}$, a qual cobre parte do Sul do Brasil, Paraguai, Uruguai e Argentina. Eventos de subsidência e mudanças no nível dos oceanos durante o Paleozóico deram origem a um sistema de seqüências, depositada no intervalo de tempo que vai do Ordoviciano ao Cretáceo Superior e apresentando inconformidades regionais (Milani, 2003).

O material aqui estudado é proveniente do Afloramento Morro Papaléo (lat. 30¹8'26”S, long. $51^{\circ} 38^{\prime} 20^{\prime} \mathrm{W}$ ), situado a $8 \mathrm{~km}$ a noroeste da sede do município de Mariana Pimentel, Rio Grande do Sul (Fig. 1). A localidade corresponde a uma seção exposta em grande parte pelas atividades de uma antiga mina a céu aberto criada para exploração de caulim pelo município de Mariana Pimentel.

De acordo com Paim et al. (1983), os pacotes sedimentares aflorantes na localidade são relacionados ao Subgrupo Itararé e à Formação Rio Bonito, perfazem uma espessura de aproximadamente $50 \mathrm{~m}$, e jazem de forma discordante sobre rochas graníticas, riolíticas e magmatíticas do embasamento.

Iannuzzi et al. (2006), identificaram dois grandes conjuntos distintos de litofaciologias para toda a sequência aflorante no Morro do Papaléo: a porção basal da seção (Subgrupo Itararé) dominada por siltitos e arenitos finos, por vezes ritmíticos, os quais são cobertos por uma capa de arenito médio; e a porção superior (Formação Rio Bonito) constituída por paraconglomerados e arenitos médios a grossos, com pacotes de pelitos carbonosos e, 


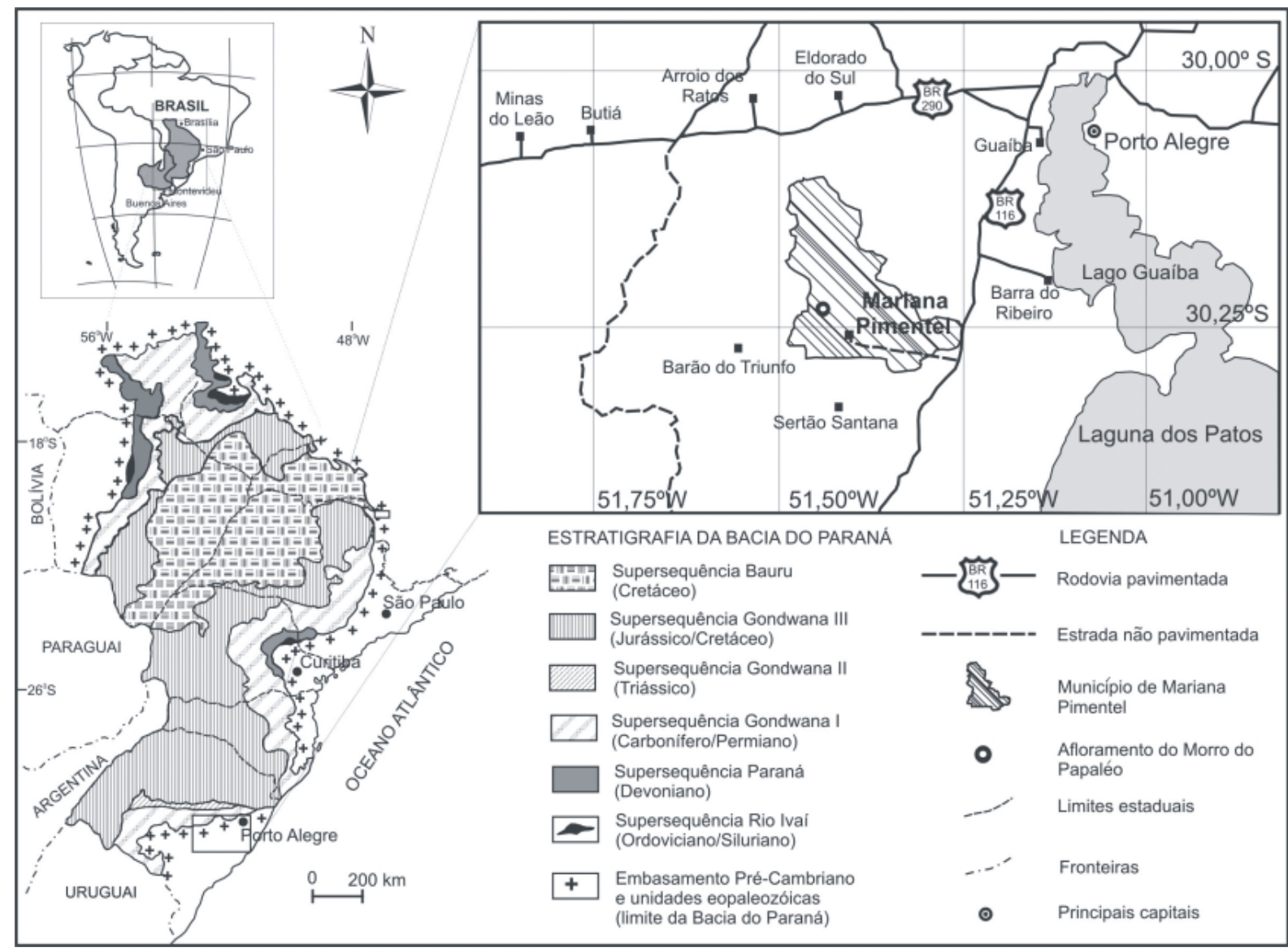

Figura 1: Mapa geológico simplificado da Bacia do Paraná com a localização do Afloramento Morro Papaléo [retirado de Smaniotto et al. (2006), Fig. 1, p. 312].

Figure 1: Simplified geological map of the Paraná Basin in Brazil with the locations of the Morro Papaléo Outcrop [adapted from Smaniotto et al. (2006), Fig. 1, p.312].

subordinadamente, ritmitos e arenitos (Fig. 2). Estes mesmos autores relatam que os diferentes níveis apresentam uma composição fitofossilífera bastante variada com elementos megaflorísticos e palinomorfos. Os elementos megaflorísticos são bastante conhecidos, sendo dominados por folhas, raízes, caules, sementes e estruturas reprodutivas de diversos grupos como licófitas, filicófitas, esfenófitas, cordaites, ginkgo e glossopterídeas (Corrêa da Silva, 1978; CazzuloKlepzig et al., 1980; Pasqualini et al., 1986; GuerraSommer \& Cazzulo-Klepzig, 1993; Vieira \& Iannuzzi, 2000; Iannuzzi et al., 2006; Tybusch \& Iannuzzi, 2008; Souza \& Iannuzzi, 2007, 2009).

Análises palinológicas detalhadas de níveis pelíticos da localidade foram conduzidas por Burjack et al. (1982); Marques-Toigo (1991); Souza \& Marques-Toigo (2003; 2005) e Smaniotto et al. (2006) mostrando que o conteúdo palinológico do Afloramento Morro Papaléo representa um importante registro paleoambiental.

Smaniotto et al. (2006) e Iannuzzi et al. (2006), identificaram a presença de palinomorfos do microplanctôn e, através de relações percentuais entre os grupos da matéria orgânica particulada, interpretaram o conjunto dos palinomorfos como sendo indicativo de um ambiente transicional com influência marinha para a porção mais basal do Subgrupo Itararé do afloramento. Já para os depósitos carbonosos do topo da sequência sedimentar da Formação Rio Bonito os mesmos autores estabeleceram um ambiente lacustre ou pantanoso.

Iannuzzi et al. (2006) propõem a divisão do Afloramento em oito níveis fossilíferos (N1 a N8), estando o conteúdo megaflorístico relacionado aos níveis N2, onde ocorrem gêneros como Botrychiopsis, Glossopteris, Gangamopteris, Cordaites, Samaropsis e Cordaicarpus; N3, com a presença de Gangamopteris, Cordaites, Chiropteis e Samaropsis; N4, com Samaropsis, Cordaicarpus, Gangamopteris, Glossopteris e Cordaites e; N7 e N8 com Gangamopteris, Glossopteris, Ginkgophytopsis, Kawizophyllum, Cordaites, Brasilodendron, Cyclodendron, Pecopteris, Sphenopteris, Neomariopteris, Asterotheca, Samaropsis e Cordaicarpus.

Os demais níveis (N1, N5 e N6) são aqueles de onde foi resgatada a palinoflora descrita no trabalho de Smaniotto et al. (2006).

Souza \& Marques-Toigo $(2003,2005)$ e Iannuzzi et al. (2006) atribuem uma idade correspondente ao Asseliano/Sakmariano com base no conteúdo 


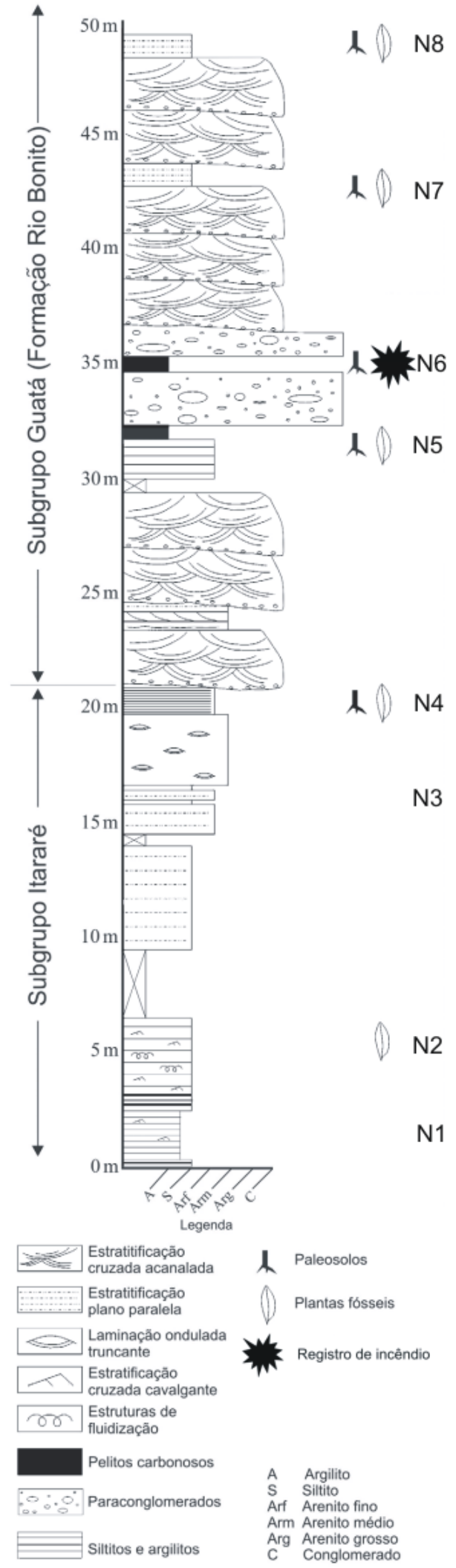

Figura 2: Seção estratigráfica do Afloramento Morro Papaléo, com indicação dos níveis com ocorrência de registros megaflorísticos, de carvão vegetal macroscópico (registro de incêndios) e dos níveis N1 a N8, descritos por Iannuzzi et al. (2006) [adaptado de Smaniotto et al. (2006), Fig. 2, p.313].

Figure 2: Stratigraphic section of Morro Papaléo Outcrop, with indication of the levels with megafloristic remains, macroscopic charcoal (evidence for wildfire) and levels N1 to N8 described by Iannuzzi et al. (2006) [adapted from Smaniotto et al. (2006), Fig.

$$
\text { 2, p. 313]. }
$$

paleopalinológico e paleobotânico para a seção aflorante no Morro Papaléo.

\section{MATERIAL E MÉTODOS}

Em trabalho de campo realizado no Afloramento Morro Papaléo foram resgatados fragmentos bem preservados de carvão vegetal macroscópico, de tamanho (comprimento) que variam de 0,3 a $28,0 \mathrm{~cm}$, inseridos em pelitos carbonosos do nível fitofossilífero N6 descrito por Iannuzzi et al. (2006), correspondente, também, a parte do nível NI de Smaniotto et al. (2006). Este material está depositados na coleção do Setor de Botânica e Paleobotânica do MCN/UNIVATES sob a sigla $\mathrm{PbU}$ (PbU 616 a $\mathrm{PbU}$ 631). Os demais níveis do afloramento, fitofossilíferos ou não, se apresentaram estéreis para fragmentos de carvão vegetal macroscópico (Fig. 2).

Com auxílio de estereomicroscópio (Modelo Leica MS5, aumentos 10 - 40 vezes) os fragmentos que apresentavam características típicas de carvão vegetal macroscópico [sensu Scott, (2010) - "coloração preto-listrada" e "brilho levemente lustroso/sedoso"] foram extraídos de forma mecânica (com utilização de uma pinça) do sedimento, tendo sido selecionadas 40 amostras.

Os espécimes não foram submetidos a processos de remoção de minerais por meio químico devido à sua fragilidade. Os material foi montado para análise em stubs com LeitC (Plano) e posteriormente examinado sob Microscópio Eletrônico de Varredura (MEV - JEOL JSM 6490 LV) no Senckenberg Forschungsinstitut und Naturmuseum, Frankfurt, Alemanha, possibilitando a observação de "detalhes anatômicos preservados" [característica necessária para a confirmação de que eram carvão vegetal macroscópico sensu Scott (2010)].

\section{RESULTADOS}

\section{Anatomia dos fragmentos de carvão vegetal macroscópico:}

Os fragmentos de carvão vegetal macroscópico estudados são visíveis a olho nu, com dimensões que variam entre $0,3-28,0 \mathrm{~cm}$ de comprimento, 0,2-14,0 $\mathrm{cm}$ de largura e 0,1-3,0 cm de espessura; além disso, as amostras apresentam bordos retos ou denteados, não estando desgastados (Fig. 3).

$\mathrm{Na}$ análise em microscópio eletrônico de varredura foi possível identificar que os tecidos dos lenhos foram macerados (Fig. 4A e B), provavelmente comprimidos durante o processo diagenético, o que impossibilitou a observação de características histológicas completas que permitissem uma conexão taxonômica específica.

Todavia, foi possível identificar dois morfotipos de lenhos, aqui chamados de PP1 e PP1 (PP para Papaléo), os quais, apesar de estarem associados a amostras diferentes, podem representar estágios de crescimento de uma mesma planta. 


\section{LENHO MORFOTIPO PP1:}

Lenho picnoxílico secundário apresentado traqueídeos em plano longitudinal radial com 28 - 32 $\mu \mathrm{m}$ de largura. Os traqueídeos exibem pontoações uni (Fig. 4C) ou biseriadas (Fig. 4D). As aberturas das pontoações seus e bordos variam de circulares a ovais com 2 - 4 um de diâmetro. Quando biseriadas, as pontoações estão dispostas de forma diagonal (Fig. 4E). As paredes celulares estão homogeneizadas e têm $1,6-2,0 \mu \mathrm{m}$ de espessura (Fig. 4F). Feixes, traços foliares, anéis de crescimento não visíveis.

\section{Lenho Morfotipo PP2:}

Lenho picnoxílico secundário em corte longitudinal tangencial, sem pontoações visíveis. Presença de raios desgastes, evidenciados pelos bordos retos ou denteados do material aqui avaliado, além de seu tamanho, são os indicativos utilizados para identificar o grau de transporte do material. Conforme descrito, o material registrado para o Afloramento Morro do Papaléo é de tamanho considerável quando comparado a outros registros da Bacia do Paraná (Jasper et al., 2008; 2011a; 2011b) e não apresenta bordos desgastados, de forma que uma deposição autóctone/parautóctone pode ser inferida.

Com base nas características observadas nos dois morfotipos de lenho é possível associá-los a gimnospermas com pontoações dos traqueídeos e raios do tipo araucarióide. Todavia, não foi possível realizar uma associação taxonômica restritiva, até nível de espécie, por exemplo. Além disso, ambas

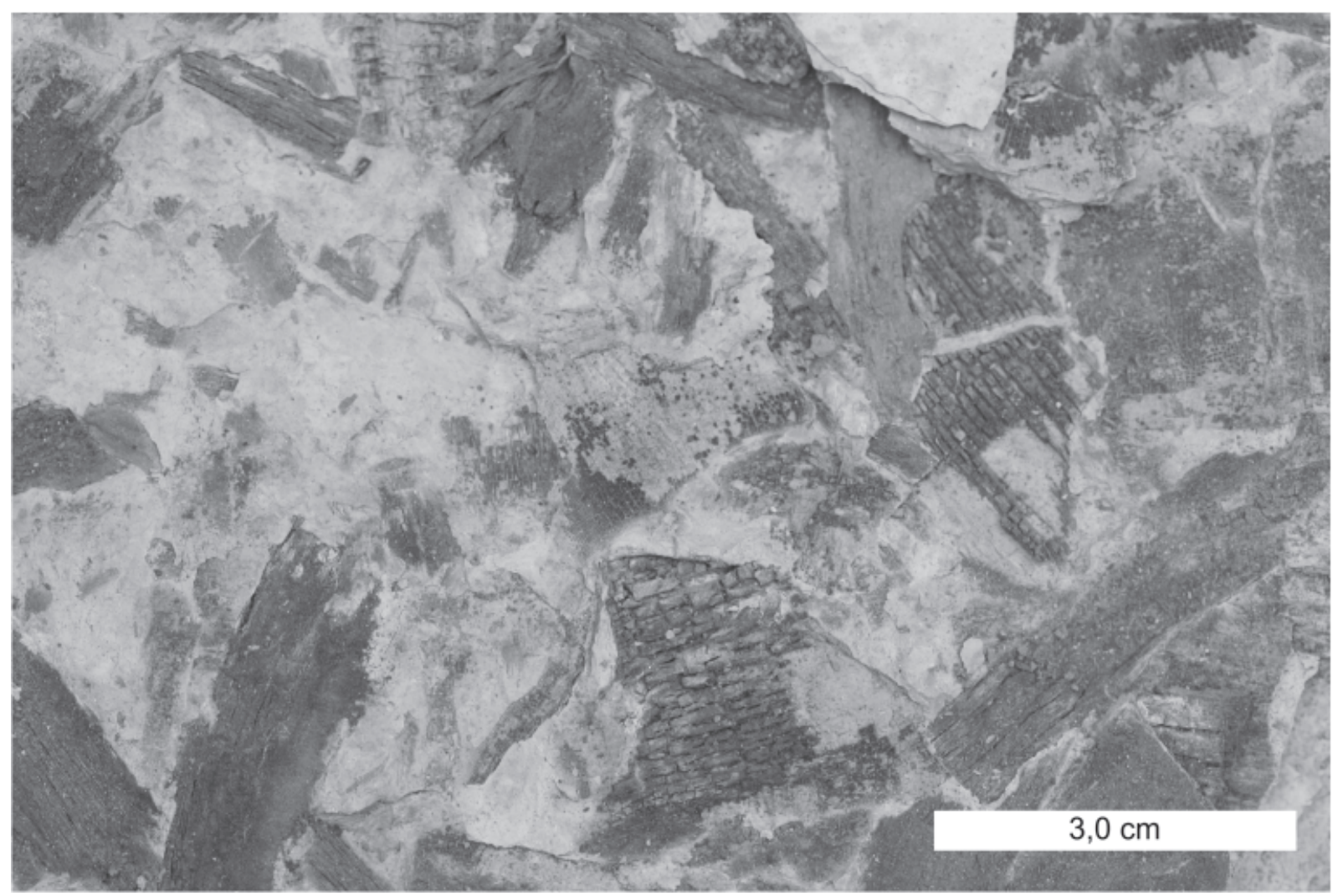

Figura 3: Amostra de mão (PbU 624) demonstrando a distribuição aleatória e a fragmentação dos carvões vegetais macroscópicos do Afloramento Morro Papaléo.

Figure 3: Hand sample (PbU 624) showing the random distribution and fragmentation of Morro Papaléo Outcrop macroscopic charcoal.

formados por células parenquimatosas com $12-15$ $\mu \mathrm{m}$ de largura e $14-20 \mu \mathrm{m}$ de altura (Fig. 4G). Raios uniseriados com $6-8$ células de altura (Fig. 4H). Feixes, traços foliares, anéis de crescimento não visíveis.

\section{DISCUSSÃO}

Uhl et al. (2003) argumentam que processos de deposição e transporte são, muitas vezes, responsáveis pela degradação dos tecidos em amostras de carvão vegetal macroscópico, de forma que a ausência de as formas podem, ainda, pertencer ao mesmo táxon, correspondendo ou a seus distintos estágios de desenvolvimento ou mesmo a planos distintos de preservação. Porém, como as amostras que originaram a descrição dos dois morfotipos aqui sugeridos não se encontravam em conexão orgânica, não é possível especular neste sentido.

Até o momento, apenas Jasper et al. (2007a, 2008; 2011a, 2011b) descreveram características anatômicas mais detalhadas de carvão vegetal macroscópico proveniente do Paleozóico Superior da Bacia do Paraná. 

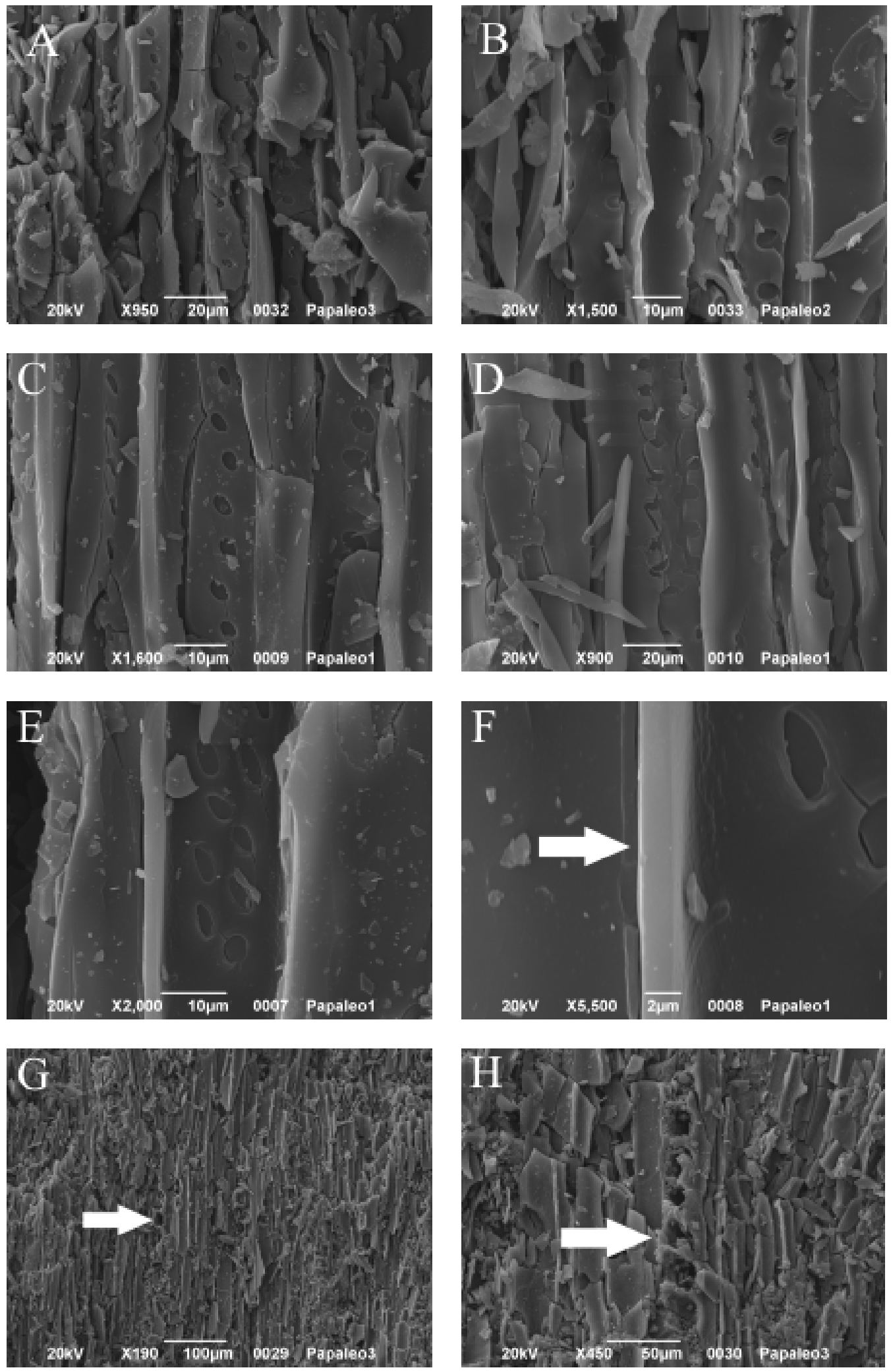

Figura 4: Imagens em MEV dos exemplares de carvão vegetal macroscópico do Afloramento Morro Papaléo: A) fragmento de lenho do morfotipo PP1 com traqueídes macerados; B) fragmento de lenho do morfotipo PP1 com traqueídes macerados; C) traqueídes exibindo pontoações unisseriadas (morfotipo PP1); D) traqueídes exibindo pontoações bisseriadas (morfotipo PP1); E) traqueíde exibindo a

distribuição diagonal das pontoações biseriadas (morfotipo PP1); F) detalhe de parede celular homogeneizada de traqueides [seta (morfotipo PP1)]; G) fragmento de lenho do morfotipo PP2 com presença de raios formados por células parenquimatosas (seta); H) detalhe da Figura $3 G$ destacando um raio [seta (morfotipo PP2)].

Figure 4: SEM images of the macroscopic charcoal samples from the Morro Papaléo Outcrop: A) wood fragment of morphotype PP1 with macerated tracheids; B) wood fragment of morphotype PP1 with macerated tracheids; $C)$ wood fragment exhibiting uniseriate bordered pitting (morphotype PP1); D) wood fragment exhibiting biseriate bordered pitting (morphotype PP1); E) tracheid exhibiting alternate distribution of the pitting; $F$ ) detail of the homogenized cell walls [arrow (morphotype PP1)]; G) wood fragment of morphotype PP2 with parenchymatic ray cells (arrow); H) detail of Figure $3 G$ showing a ray [arrow (morphotype PP2)]. 
Todavia, diferenças nas dimensões, características das pontoações (uniseriadas, biseriadas ou triseriadas) e estrutura dos raios, demonstraram que os morfotipos aqui descritos são diferentes daqueles descritos por Jasper et al. (2008, 2011b).

Assim como o Lenho Tipo 2 (Wood Type 2) de Jasper et al. (2008), a presença de pontoações e raios do tipo araucarióide corresponde às características do material associado aos lenhos tipo Dadoxylon. Apesar deste morfogênero ser, provavelmente, o melhor conhecido entre os lenhos do Paleozóico Superior, Dadoxylon é um nome inválido, sinonimizado a Agathoxylon por Bamford \& Philippe (2001) para designar os lenhos gimnospérmicos com anatomia araucarióde do Paleozóico Superior.

Com base nos caracteres aqui apresentados, uma associação genérica precisa torna-se bastante difícil, todavia, é possível inferir que os lenhos aqui descritos podem pertencer ao morfogênero Agathoxylon, o qual pode definir, ainda, diferentes táxons.

Além da amplitude de associações possíveis para o morfogênero, em termos de análises paleoecológicas, Agathoxylon não é um bom indicador. Falcon-Lang \& Bashforth (2005), definem que o táxon (referido ainda como Dadoxylon pelos autores) ocorre em ambientes diversos, desde áreas mais pantanosas, passando por planícies aluviais e chegando a encostas de montanhas localizadas em porções mais interioranas dos continentes.

No caso do Afloramento Morro Papaléo, reconstituições paleoecológicas como as de Pasqualini et al. (1986), Guerra-Sommer \& Cazzulo-Klepzig (2000a), estabelecem um ambiente lêntico para o seu sistema deposicional. Posteriormente, Smaniotto et al. (2006) e Iannuzzi et al. (2006), propõem uma influência marinha para este sistema no que tange à seção aflorante associada ao Subgrupo Itararé, e um ambiente lacustre a pantanoso para a porção da Formação Rio Bonito.

De forma mais específica para o nível fitofossilífero de onde foram resgatados os fragmentos de carvão vegetal macroscópico aqui estudados, Smaniotto et al. (2006) conduziram estudos palinológicos que confirmaram a presença de esporos (Brevitriletes, Lundbladispora, Punctatisporites), grãos de pólen (Cannanoropolis, Illinites, Vittatina), algas (Tetraporina, Quadrisporites) e fungos (Portalites), o que permitiu uma interpretação de ambiente pantanoso para a deposição.

Destaca-se, ainda, que os lenhos do gênero Agathoxylon foram elementos comuns tanto no Hemisfério Norte quanto no Hemisfério Sul durante o Paleozóico Superior, adquirindo características cosmopolitas. De acordo com Philippe et al. (2004), isto indica que o gênero teve condições de sobreviver nas diferentes zonas climáticas do=Planeta, não sendo, portanto, um bom indicador paleoclimático. Todavia, a presença de Botrychiopsis plantiana nos níveis mais basais da associação estudada permitiu a Jasper et al. (2003, 2007a) inferir um clima frio para a porção basal do afloramento (Subgrupo Itararé), que se tornaria mais ameno na porção superior da sucessão.
Conforme definido por Scott \& Glasspool (2007) o registro de fragmentos de carvão vegetal macroscópico é indicativo direto da ocorrência de incêndios vegetacionais. Além disso, Samiotto et al. (2006) comprova a abundante presença de fitoclastos opacos, angulosos e mal selecionados no mesmo nível de onde provém o material aqui estudado, de forma que é possível associar este domínio de fitoclastos definidos com base em critérios de análise de palinofácies à manifestação de paleoincêndios nas áreas de entorno ou no próprio local de deposição.

De acordo com Guerra-Sommer \& CazzuloKlepzig (2000b), com a retração das capas de gelo, a sedimentação glaciogênica do Subgrupo Itararé foi substituída por sistemas fluviodeltáicos e estuarinos da Formação Rio Bonito.

Para Flannigan et al. (2009) os ambientes pantanosos não são os mais propícios para a manifestação de eventos de incêndios vegetacionais, apesar da presença de uma grande quantidade de combustível nessas áreas, já que o ambiente anóxico nas porções mais próximas ao solo impediria que o fogo se alastrasse. Todavia, Beckage \& Platt (2003) defendem que, em havendo uma estação seca que dure no mínimo dois meses, mesmo ambientes pantonosos podem estar sujeitos a incêndios. Assim, considerando a ausência de indícios de transporte no material aqui estudado, é possível inferir que os paleoincêndios vegetacionais responsáveis por sua formação tenham atingido a turfeira que originou o nível de carvão.

Outro aspecto importante observado nas amostras é o grau de fusão (homogeneização) das paredes celulares. Com base nas análises experimentais de Jones \& Chaloner (1991), o mesmo indica uma temperatura de queima variando entre $340^{\circ} \mathrm{C}$ e $600^{\circ} \mathrm{C}$, o que está de acordo com as temperaturas das frentes de queima nas porções basais áreas de turfeiras atuais (Rein et al., 2008).

Quando se questiona a fonte da ignição dos paleoincêndios registrados no Afloramento Morro do Papaléo, é importante considerar que para associações paleoflorísticas próximas de idade semelhante, como é o caso do Afloramento Quitéria (Jasper et al., 2008), da Mina do Faxinal (Jasper et al., 2011b), da Mina Leão-Butiá (Jasper et al., 2011a) e da Mina de Candiota (Jasper et al., 2011a), o vulcanismo foi indicado como possível responsável. De acordo com Guerra-Sommer et al. (2008), existem evidências de atividades vulcânicas dispersas em diferentes sucessões formadoras de carvão na porção sul do Brasil. Nas Minas de Candiota e do Faxinal são encontrados, por exemplo, níveis contínuos de sedimentos vulcânicos entre os níveis de carvão (Formoso et al., 1999; Guerra-Sommer et al., 2008). Para o Afloramento Quitéria, Jasper et al. (2006) confirmam a presença de fragmentos de vidro vulcânico não retrabalhado em nível de paraconglomerado, o que também confirma existência de atividades vulcânicas ocorridas durante a deposição da porção basal da associação. 
Apesar de não haver indícios diretos de vulcanismo descritos para o Afloramento Morro do Papaléo, e de haverem outras formas de ignição conhecidas (como a ocorrência de tempestades elétricas, por exemplo), a confirmação da ocorrência de paleoincêndios no sistema deposicional formador da associação ali registrada permite, ao menos, pensar em uma correlação entre os eventos de ignição ocorridos nos sistemas próximos e os que iniciaram os registrados na área de estudo, provavelmente concentrados em estações secas com duração superior a dois meses.

Correlacionando os dados aqui apresentados e os registros de carvão vegetal macroscópico anteriormente citados para outras localidades com associações paleoflorísticas semelhantes, é possível, também, sugerir uma ocorrência simultânea dos paleoincêndios em um período relativamente curto de tempo (Asseliano/Sakmariano) na porção sul da Bacia do Paraná, associados especificamente aos sistemas formadores de carvão ali existentes.

Por fim, o presente estudo, associado a estudos prévios, como os de Jasper et al. (2007a, 2008; 2011b) e Uhl et al. (2008), demonstra que o registros de carvão vegetal macroscópico é frequente nas sequências sedimentares paleozóicas da Bacia do Paraná, confirmando que paleoincêndios vegetacionais formam comuns neste período, inclusive nos sistemas formadores dos níveis de carvão. Desta forma, as grandes concentrações de inertinita nos carvões permianos da Bacia do Paraná podem ter se originado pela manifestação deste tipo de evento, discordando de estudos anteriores como os de White (1925), Jurasky (1929), Schopf (1975) e Taylor et al. $(1989,1998)$ que desvinculavam os dois fatos.

\section{CONCLUSÕES}

A partir das evidências aqui apresentadas, é possível concluir que:

(1) Está confirmada, através do registro de carvão vegetal macroscópico, a ocorrência de Paleoincêndios vegetacionais em pelo menos um dos ambientes associados à deposição registrada no Afloramento Morro Papaléo, Bacia do Paraná;

(2) Os lenhos que compõem os carvões vegetais macroscópicos analisados são de origem gimnospérmica, podendo estar associados ao morfogênero Agathoxylon;

(3) A ausência de bordos desgastados e a característica macroscópica $(>2,0 \mathrm{~mm})$ dos fragmentos estudados atesta uma deposição autóctone/parautóctone para o material, confirmando que a turfeira na qual eles foram preservados estava sujeita a ocorrência de paleoincêndios vegetacionais;

(4) A ocorrência de incêndios vegetacionais indica que o ambiente (ao menos aquele registrado no nível estudado) estava sujeito a uma estação seca com duração mínima de dois meses;

(5) Tomando como base localidades próximas, é possível inferir que uma das formas de ignição para os paleoincêndios vegetacionais registrados no Afloramento Morro do Papaléo, foram os processos vulcânicos disseminados nos sistemas contemporâneos.

\section{AGRADECIMENTOS}

Os autores agradecem a Claudia Franz, Senckenberg Forschungsinstitut und Naturmuseum de Frankfurt, pelo auxílio técnico na operação do MEV. A. Jasper, J. Manfroi e E. Ost e N. T. G. Machado agradecem o suporte financeiro da UNIVATES, FAPERGS e CNPq. Os autores agradecem, ainda, aos revisores anônimos pelas suas importantes contribuições.

\section{REFERÊNCIAS}

BAMFORD M.K. \& PHILIPPE M. 2001. Jurassic-Early Cretaceous Gondwanan homoxylous woods: a nomenclatural revision of the genera with taxonomic notes. Review of Palaeobotany and Palynology, 113: 287-297.

BECKGE B. \& PLANT W.J. 2003. Predicting severe wildfire years in the Florida Everglades. Frontiers in Ecology and the Environment, 1: 235-239.

BOND W.J. \& KEELEY J.E. 2005. Fire as a global "herbivore": the ecology and evolution of flammable ecosystems. Trends in Ecology and Evolution, 20(7): 387-394.

BOWMAN D.M.J.S., BALCH J.K., ARTAXO P., BOND W.J., CARLSON J.M., COCHRANE M.A., D'ANTONIO C.M., DEFRIES R.S., DOYLE J.C., HARRISON S.P., JOHNSTON F.H., KEELEY J.E., KRAWCHUK M.A., KULL C.A., MARSTON J.B., MORITZ M.A., PRENTICE I.C., ROOS C.I., SCOTT A.C., SWETNAM T.W., VAN DER WERF G.R., PYNE S.J. 2009. Fire in the Earth System. Science, 324: 481-484.

BURJACK M.I.A., CAZZULO-KLEPZIG M., DIAS-FABRICIO M.A., GUERRA-SOMMER M., MARQUES-TOIGO M., PAIM P.S., LAVINA E.L. 1982. Perfil paleoecológico do Afloramento Morro do Papaléo, Permiano Inferior da Bacia do Paraná, Rio Grande do Sul, Brasil. In: CONGRESSO BRASILEIRO DE GEOLOGIA, 32, 1982. Anais, Salvador, SBG, p. 1260-1270.

CAZZULO-KLEPZIG M., GUERRA-SOMMER M., BOSSI G.E. 1980. Revisão Fitoestratigráfica do Grupo Itararé no RS: Acampamento Velho, Cambaí Grande, Budó e Morro do Papaléo. Boletim do Instituto de Geociências da USP, 11: 55-76.

CORRÊA DA SILVA Z.C. \& ARRONDO O.G. 1977. Tafoflora Permiana de Mariana Pimentel, Município de Guaíba, Rio Grande do Sul, Brasil. Revista Pesquisas, 7: 27-44.

CORRÊA DA SILVA Z.C. 1978. Observações sobre o Grupo Tubarão no Rio Grande do Sul com especial destaque à estratigrafia da Formação Itararé. Revista Pesquisas, 9: 9-61.

DIMICHELE W.A., HOOK R.W., NELSON W.J., CHANEY D.S. 2004. An unusual Middle Permian flora from the Blaine Formation (Pease River Group: Leonardian-Guadalupian Series) of King County, West Texas. Journal of Paleontology, 78: 765-782.

EDWARDS D. \& AXE L. 2004. Anatomical evidence in the detection of the earliest wildfire. Palaios, 19: 113-128.

FALCON-LANG H.J. 1998. The impact of wildfire on an Early Carboniferous coastal environment, North Mayo, I r e l a n d. Palaeogeography, Palaeoclimatology, Palaeoecology, 139: 121-138.

FALCON-LANG H.J. \& BASHFORTH A.R. 2005. Morphology, anatomy and upland ecology of large cordaitalean trees from the Middle Pennsylvanian of Newfoundland. Review of Palaeobotany and Palynology, 135: 223-243.

FALCON-LANG H.J. \& SCOTT A.C. 2000. Upland ecology of some Late Carboniferous cordaitalean trees from Nova Scotia and England. Palaeogeography, Palaeoclimatology, Palaeoecology, 156: $225-242$ 
FLANNIGAN M.D., KRAWCHUK M.A., GROOT W.J. DE, WOTTON B.M., GOWMAN L.M. 2009. Implications of changing climate for global wildland fire. International Journal of Wildland Fire, 18: 483-507.

FORMOSO M.L.L. 1968. Alguns aspectos geológicos e tecnológicos das principais ocorrências de argilas no Rio Grande do Sul. Revista Cerâmica, 14(53): 1-13.

GLASSPOOL I.J. 2000. A major fire event recorded in the mesofossils and petrology of the Late Permian, Lower Whybrow coal seam, Sydney Basin, Australia. Palaeogeography, Palaeoclimatology, Palaeoecology, 164: 373-396.

GLASSPOOL I.J., EDWAEDS D., AXE L. 2004. Charcoal in the Silurian as evidence for the earliest wildfire. Geology, 32: 381-383.

GUERRA-SOMMER M. \& CAZZULO-KLEPZIG M. 1993. Biostratigraphy of the Southern Brazilian Neopaleozoic Gondwana Sequence: a preliminary palaeobotanical approach. In: INTERNATIONAL CONGRÉS DE LA STRATIGRAPHIE ET GÉOLOGIE DU CARBONIFÉRE ET PERMIEN, 12, 1991. Buenos Aires, Comptes rendus, 2: 61-72.

GUERRA-SOMMER M. \& CAZZULO-KLEPZIG M. 2000a. As floras gonduânicas do Paleozóico Superior do Rio Grande do Sul. In: HOLZ M. \& ROS L.F. DE (eds.) Paleontologia do Rio Grande do Sul, Editora da UFRGS, p. 67-84.

GUERRA-SOMMER M. \& CAZZULO-KLEPZIG M. 2000b. Early Permian paleofloras from southern Brazilian Gondwana: a paleoclimatic approach. Revista Brasileira de Geociências, 30(3): 486-490.

GUERRA-SOMMER M., CAZZULO-KLEPZIG M., SANTOS J.O.S., HARTMANN L. A., KETZER J.M., FORMOSO M.L.L. 2008. Radiometric age determination of tonstein and stratigraphic constrains for the Lower Permian coal succession in southern Paraná Basin, Brazil. International Journal of Coal Geology, 74: 13-27.

IANNUZZI R., SCHERER C.M.S., SOUZA P.A., HOLZ M., CARAVACA G., ADAMI-RODRIGUES K., TYBUSCH G.P., SOUZA J.M., SMANIOTTO L.P., FISCHER T.V., SILVEIRA A.S., LYKAWKA R., BOARDMAN D.R., BARBOZA E.G 2006. Afloramento Morro do Papaléo, Mariana Pimentel, RS: Registro ímpar da sucessão pós-glacial do Paleozóico da Bacia do Paraná. In: WINGE M., SCHOBBENHAUS C., BERBERT-BORN M., QUEIROZ E.T., CAMPOS D.A., SOUZA C.R.G., FERNANDES A.C.S. (eds.) Sítios Geológicos e Paleontológicos do Brasil, Volume II, CPRM, p. 321-336.

JASPER A., GUERRA-SOMMER M., CAZZULO-KLEPZIG M., MENEGAT R. 2003. The Botrychiopsis genus and its biostratigraphic implications in Southern Paraná Basin. Anais da Academia Brasileira de Ciência, 75(4): 513-535.

JASPER A., MENEGAT R., GUERRA-SOMMER M., CAZZULOKLEPZIG M., SOUZA, P.A. 2006. Depositional cyclicity and paleoecological variability in an outcrop of Rio Bonito formation, Early Permian, Paraná Basin, Rio Grande do Sul, Brazil. Journal of South American Earth Science, 21: 276-293.

JASPER A., GUERRA-SOMMER M., CAZZULO-KLPEZIG M., IANNUZZI R. 2007a. Biostratigraphic and paleoclimatic significance of Botrychiopsis fronds in Gondwana realm. In: WONG T.H.E. (ed.). Proceedings of the XVth International Congress on Carboniferous and Permian Stratigraphy, EditaKNAW, p. 379-388.

JASPER A., GUERRA-SOMMER M., UHL D., SALVI J., KAUFFMANN M., OSTEKAMP I.C., GONÇALVES C.V. 2007b. A ocorrência de incêndios vegetacionais durante o Paleozóico Superior da Bacia do Paraná. In: CARVALHO I., CASSAB R.C.T., SCHWANKE C., CARVALHO M.A., FERNANDES A.C.S., RODRIGUES M.A.C., CARVALHO M.S.S., ARAI M., OLIVEIRA M.E.Q. (eds.). Paleontologia: Cenários de Vida, Editora Interciência, volume 1, p.14-25.

JASPER A., UHL D., GUERRA-SOMMER M., MOSBRUGGER V. 2008. Palaeobotanical evidence of wildfires in the Late Palaeozoic of South America - Early Permian, Rio Bonito Formation, Paraná Basin, Rio Grande do Sul, Brazil. Journal of South American Earth Sciences, 26: 435-444.

JASPER A., UHL D., MACHADO N.T.G., GONÇALVES C.V., GUERRA-SOMMER M., CAZZULO-KLEPZIG M. 2009
Late Paleozoic wildfires in the South American part of the Gondwana Realm. In: INTERNATIONAL LATAINAMERIKAKOLLOQUIUM, 2009. Abstracts, Göttingen, p. 146-147.

JASPER A, UHL D., GUERRA-SOMMER M., BERNARDESDE-OLIVEIRA M.E.C., MACHADO N.T.G. 2011a. Upper Paleozoic remains from South America: multiple evidences of fire events in the coal bearing strata of the Paraná Basin, Brazil. Palaeogeography, Palaeoclimatology, Palaeoecology, 306: $205-218$.

JASPER A., UHL D., GUERRA-SOMMER M., HAMAD A.M.B.A., MACHADO N.T.G. 2011b. Paleowildfires in Gondwana Realm: remains from a tonstein in the Faxinal Coalfield, a Lower Permian coal succession southern Paraná Basin, Brazil. Anais da Academia Brasileira de Ciências, 83(2): 471-481.

JURASKY K.A. 1929. Neue Untersuchungen und Gedanken zur Entstehung Fossiler Holzkohle. In: STUTZER O. (ed.). Fusit-Vorkommen, Entstehung und Praktische Bedeutung der Faserkohle: Schriften aus dem Gebiet der Brennstoff Geologie, Verlag von Ferdinand Enke, Stuttgart, Heft, 2, p. 23-41.

JONES T.P. \& CHALONER W.G. 1991. Fossil charcoal, its recognition and palaeoatmospheric significance. Palaeogeography Palaeoclimatology, Palaeoecology, 97: 39-50.

LAVINA E.L., LOPES R.C., FACCINI U.F., RIBEIRO H.J.P.S., SCHULTZ C.L. 1992. O sistema Permo-Triássico da Bacia do Paraná no Estado do RS. In: CONGRESSO BRASILEIRO DE PALEONTOLOGIA, 13, 1993. Anais, São Leopoldo, UNISINOS, p. 32.

MACDONALD G.M., LARSEN C.P.S., SZEICS J.M., MOSER K.A. 1991. The reconstruction of boreal forest fire history from lake sediments: a comparison of coal, pollen, sedimentological, and geochemical indexes. Quaternary Science Reviews, 10: 53-71.

MARQUES-TOIGO M. 1991. Palynobioestratigraphy of the Southern Brazilian Neopaleozoic Gondwana sequence. In: INTERNACIONAL GONDWANA SYMPOSIUM, 7, 1988. Proceedings, São Paulo, p. 503-515.

MILANI E.J. 2003. Estratigrafia da Bacia do Paraná - Algumas considerações metodológicas. In: ENCONTRO S O B R E ESTRATIGRAFIA DO RIO GRANDE DO SUL: ESCUDO E BACIAS, 2003. Anais, Porto Alegre, p. 18-22.

PAIM P.S.G., PICCOLI A.E.M., SARTURI J.A.D., MUNARO P., HOLZ M., GRANITOFF W. 1983. Evolução paleogeográfica do Supergrupo Tubarão na área de Mariana Pimentel-Faxinal, Guaíba, RS.In: SIMPÓSIO SUL-BRASILEIRODE GEOLOGIA, 1983. Atas, Porto Alegre, SBG, p. 121-134.

PASQUALINI M., CUNHA A.S., GUERRA-SOMMER M., PICCOLI A.E.M. 1986. Análise paleoecológica de seqüências paleoflorísticas na área de Mariana Pimentel-Guaíba, RS. In: CONGRESSO BRASILEIRO DE GEOLOGIA, 34, 1986. Anais, Goiânia, SBG, p. 556-569.

PHILIPPE M., BAMFORD M., MCLOGHLIN S., ALVES L.S.R., FALCON-LANG H.J., GNAEDINGER S., ORRONE E.G., POLE M., RAJANIKANTHA., SHOEMAKER R.E., TORRES T., ZAMUNER A. 2004. Biogeographic analysis of JurassicEarly Cretaceous Wood assemblages from Gondwana. Review of Palaeobotany and Palynology, 129: 141-173.

PICCOLI A.E.M., PAIM P.S.G., SARTURII J.A.D., HOLZ M., MUNARD P., GRANITOFF W. 1983. Geologia do Supergrupo Itararé na região de Mariana Pimentel-Faxinal, Guaíba, RS. In: SIMPÓSIO SUL-BRASILEIRO DE GEOLOGIA, 1, 1983. Atas, Porto Alegre, SBG, p. 135-152.

PRESTON C.M., SCHMIDT M.W.I. 2006. Black (pyrogenic) carbon: a synthesis of current knowledge and uncertainties with special consideration of boreal regions. Biogeosciences, 3: 397-420.

REIN G., CLEAVER N., ASHTON C., PIRONI P., TORERO J.L. 2008. The severity of smouldering peat fires and damage to the forest soil. Catena, 74: 304-309.

ROBINSON J.M. 1989. Phanerozoic O, variation, fire, and terrestrial ecology. Palaeogeography, Palaeoclimatology, Palaeoecology, 75: 223-240.

ROBINSON J.M. 1991. Phanerozoic atmospheric reconstructions: a terrestrial perspective. Palaeogeography, Palaeoclimatology, Palaeoecology, 97: 51-62.

SANDER P.M. 1987. Taphonomy of the Lower Permian Geraldine bonebed in Archer County Texas. Palaeogeography, 
Palaeoclimatology, Palaeoecology, 61: 221-236.

SANDER P.M. \& GEE C.T. 1990. Fossil charcoal: techniques and applications. Review of Palaeobotany and Palynology, 63: 269-279.

SCHOPF J.M. 1975. Models of fossil preservation. Review of Palaeobotany and Palynology, 20: 27-53.

SCOTT A.C. 1989. Observations on the nature and origin of fusain. International Journal of Coal Geology, 12: 443-475.

SCOTT A.C. 2000. The pre-quaternary history of fire. Palaeogeography, Palaeoclimatology, Palaeoecology, 164: 281-329.

SCOTT A.C. 2001. Preservation by fire. In: Briggs, D.E.G. \& Crowther P.R. (eds.). Palaeobiology a synthesis II. Blackwell Scientific Publishing, p. 277-280.

SCOTT A.C. 2010. Charcoal recognition, taphonomy and uses in palaeoenvironmental analysis. Palaeogeography, Palaeoclimatology, Palaeoecology, 291: 11-39.

SCOTT A.C. \& DAMBLON F. 2010. Charcoal: Taphonomy and significance in geology, botany and archaeology. Palaeogeography, Palaeoclimatology, Palaeoecology, 291: 1-10.

SCOTTA.C. \& GLASSPOOL I. 2006. The Diversification of Paleozoic fire systems and fluctuation in atmospheric oxygen concentration. PNAS, 103(29): 10861-10865.

SCOTT A.C. \& GLASSPOOL I. 2007. Observations and experiments on the origin and formation of inertinite group macerals. International Journal of Coal Geology, 70: 53-66.

SCOTT A.C. \& STEA, R. 2002. Fire sweep across the Mid-Cretaceous landscape of Nova Scotia. The Geoscientist, 12(1): 3-6.

SMANIOTTO L.P., FISCHER T.V., SOUZA P.A., IANNUZZI R. 2006. Palinologia do Morro do Papaléo, Mariana Pimentel (Permiano Inferior, Bacia do Paraná), Rio Grande do Sul, Brasil. Revista Brasileira de Paleontologia, 9(3): 311-322.

SOUZA J.M. \& IANNUZZI R. 2007. Sementes do gênero Samaropsis Goeppert no Permiano Inferior da Bacia do Paraná, sul do Brasil. Revista Brasileira de Paleontologia, 10(2): 93-106.

SOUZA J.M. \& IANNUZZI T. 2009. The genus Cordaicarpus Geinitz in the Lower Permian of the Paraná Basin, Rio Grande do Sul, Brazil. Revista Brasileira de Paleontologia, 12(1): 5-16.

SOUZA P.A. \& MARQUES-TOIGO M. 2003. An overview on the palynostratigraphy of the Upper Paleozoic strata of theBrazilian Paraná Basin. Revista del Museo Argentino Ciencias Naturales, 5(2): 205-214.

SOUZA P.A. \& MARQUES-TOIGO M. 2005. Progress on the palynostratigraphy of the Permian strata in Rio Grande do Sul State, Paraná Basin, Brazil. Anais da Academia Brasileira de Ciências, 77: 353-365.

TAYLOR G.H., LIU S.Y., DIESSEL C.F.K. 1989. Thecold climate origin of inertinite-rich Gondwana coals. International Journal of Coal Geology, 11: 1-22.

TAYLOR G.H., TEICHMULLER M., DAVIS A., DIESSEL C.F.K., LITTKE R., ROBERT P. 1998. Organic Petrology. Gebruder Borntraeger, Berlin.

TYBUSCH, G.P. \& IANNUZZI R. 2008. Reavaliação taxonômica dos gêneros Gangamopteris e Rubidgea, Permiano Inferior da
Bacia do Paraná, Brasil. Revista Brasileira de Paleontologia, 11(2): 73-86.

UHL D. \& KERP H. 2003. Wildfires in the Late Palaeozoic of Central Europe - The Zechstein (Upper Permian) of NW Hesse (Germany). Palaeogeography, Palaeoclimatology, Palaeoecology, 199: 1-15.

UHL D., JASPER A., HAMAD A.M.B.A., MONTENARI M. 2008. Permian and Triassic wildfires and atmospheric o x y g e n levels. Proceedings of WSEAS Conferences - Special Issue, 13: 179-187.

UHL D., LAUSBERG S., NOLL R., STAPF K.R.G. 2004. Wildfires in the Late Palaeozoic of Central Europe - an overview of the Rotliegend (Upper Carboniferous-Lower Permian) of the Saar-Nahe Basin (SW-Germany). Palaeogeography, Palaeoclimatology, Palaeoecology, 207: 23-35.

VIEIRA C.E.L. \& IANUUZZI R. 2000. Presença de Pecopteris e Asterotheca no afloramento Morro do Papaléo, município de Mariana Pimentel, RS (Formação Rio Bonito, Eopermiano da Bacia do Paraná). Revista Pesquisas, 27: 49-64.

WANG ZI.Q. \& CHEN AN.S. 2001. Traces of arborescent lycopsids and dieback of the forest vegetation in relation to the terminal Permian mass extinction in North China. Review of Palaeobotany and Palynology, 117: 217-243.

WHITE D. 1925. Environmental conditions of deposition of coal. American Institute of Mining, Metallurgy and Engineering: Transaction, 71: 3-34. 